\title{
Individual differences in distinct components of attention are linked to anatomical variations in distinct white matter tracts
}

\author{
Sumit Niogi ${ }^{1}$, Pratik Mukherjee ${ }^{2}$, Jamshid Ghajar ${ }^{1,3}$ and Bruce D. McCandliss ${ }^{4 *}$ \\ Weill-Cornell Medical College, Cornell University, Ithaca, NY, USA \\ 2 Department of Radiology, University of California, San Francisco, CA, USA \\ 3 Brain Trauma Foundation, New York, NY, USA \\ ${ }^{4}$ Department of Psychology and Human Development, Vanderbilt University, Nashville, TN, USA
}

Edited by:

Kathleen S. Rockland, MIT, USA

Reviewed by:

Yalchin Abdullaev, University of Oregon, USA

Jean C. Augustinack, Athinoula A. Martinos Center for Biomedical

Imaging, USA

*Correspondence:

Bruce D. McCandliss, Educational Neuroscience Laboratory, Department of Psychology and Human

Development, Vanderbilt University, \#552, 230 Appleton Place, Nashville, TN 37203-5721, USA.

e-mail:bruce.mccandliss@ vanderbilt.edu
Inter-subject variations in white matter tract properties are known to correlate with individual differences in performance in cognitive domains such as attention. The specificity of such linkages, however, is largely unexplored at the level of specific component operations of attention associated with distinct anatomical networks. This study examines individual performance variation within three functional components of attention - alerting, orienting, and conflict processing - identified by the Attention Network Task (ANT), and relates each to inter-subject variation in a distinct set of white matter tract regions. Diffusion tensor imaging data collected at $3 T$ was used to calculate average fractional anisotropy within a set of individualized a priori defined regions of interest using the Reproducible Objective Quantification Scheme (ROQS) (Niogi and McCandliss, 2006; Niogi et al., 2007). Results demonstrate three functionally distinct components of attention that each correlate distinctly with three white matter tract regions. Structure-function correlations were found between alerting and the anterior limb of the internal capsule, orienting and the splenium of the corpus callosum, and conflict and the anterior corona radiata. A multiple regression/dissociation analysis demonstrated a triple dissociation between these three structure-function relationships that provided evidence of three anatomically and functionally separable networks. These results extend previous findings from functional imaging and lesion studies that suggest these three components of attention are subserved by dissociable networks, and suggest that variations in white matter tract microstructure may modulate the efficiency of these cognitive processes in highly specific ways.

Keywords: diffusion tensor imaging, fractional anisotropy, attention, orienting, conflict, internal capsule, anterior corona radiata, corpus callosum

\section{INTRODUCTION}

White matter tracts provide the anatomical connectivity essential for normal cognitive functioning that requires the integration of neural computation across spatially separated cortical regions such as attention and executive function abilities. This has potentially strong implications for understanding how variations in structural properties of white matter tracts from one person to another may systematically influence individual variations in efficiency across a wide range of cognitive domains, even within healthy individuals exhibiting no signs of neural or cognitive dysfunction. In support of this notion of a dimensional structure-function relationship between white matter tract microstructure and cognitive abilities, a rising number of diffusion tensor imaging (DTI) studies have shown that individual differences in white matter microstructure are systematically linked to individual differences in cognitive domains including reading and phonological processing (Klingberg et al., 2000; Beaulieu et al., 2005; Deutsch et al., 2005; Niogi and McCandliss, 2006; Dougherty et al., 2007; Qiu et al., 2008; Odegard et al., 2009), numeracy and mathematical abilities (Ewing-Cobbs et al., 2006; van Eimeren et al., 2008), executive attention (Olesen et al., 2003; Liston et al., 2006; Grieve et al., 2007; Niogi et al., 2008b), visual attention (Tuch et al., 2005; Madden et al., 2006), alerting (Nestor et al., 2007), and memory (Olesen et al., 2003; Niogi and McCandliss, 2006; Niogi et al., 2008b; Schiavone et al., 2009; Zahr et al., 2009). Additionally, it has been demonstrated that reaction time measures are sensitive to detecting variations in efficiency of cognitive domains (Tuch et al., 2005; Niogi et al., 2008b).

Moreover, a growing body of evidence suggests that DTI assessments taken from separate white matter tracts may correlate specifically with performance in different cognitive domains. As an example, DTI measures from two distinct white matter tracts in the same population of subjects have been found to correlate with performance in two distinct cognitive domains (Niogi and McCandliss, 2006; Niogi et al., 2008b). Niogi and McCandliss (2006) showed that within the same population correlations exist between reading ability and fractional anisotropy (FA) in a left superior-inferior fiber tract, but these measures were unrelated to a similar structure-function correlation between short term memory and FA in a frontal association tract. Similarly, double dissociation findings within a single population of normal healthy adults demonstrating structural and functional specificity were found for 
the relationships between attention and FA in the anterior corona radiata (ACR) versus long term memory formation and the uncinate fasciculus (UF) (Niogi et al., 2008b).

Such findings serve to establish that specific, separable structurefunction associations can be found across different neural networks, and variations in white matter tract properties are often closely linked to variations within these distinct cognitive domains. Such individual differences studies, however, have yet to examine whether such structure-function distinctions might also hold true for components within a specific cognitive domain. The purpose of this study is to use DTI to examine structure-function relationships of three specific cognitive processes identified as components of the general domain of attention, and to assess whether individual differences in the cognitive efficiency of each component process may be specifically linked to individual variations in microstructural properties within each of these distinct white matter tracts.

Attention is a complex cognitive domain that has been extensively investigated by employing cognitive paradigms attempting to isolate functional components as well as by employing neural studies to investigate their associated brain systems. Although many competing theories have proposed a number of potential components of attention, one highly influential approach ${ }^{1}$ has proposed that three broad functional distinctions can be made that account for a multitude of findings across cognitive studies, neuropsychological investigations, and neuroimaging studies. Posner and Petersen (1990) proposed that investigating attention in terms of three separable component functional processes - alerting, orienting, and executive function - would help integrate a host of cognitive and neural investigations. Over the last two decades, these components of attention have been linked to separable brain networks via functional neuroimaging, electrophysiology, and lesion studies (Posner and Rothbart, 2006; Posner et al., 2006; Raz and Buhle, 2006; Fan et al., 2009).

The efficiency of the individual components of the attention system proposed by Posner and Petersen can be separately measured using the Attention Network Test (ANT) (Fan et al., 2002). The ANT is a reaction time task that measures the latency to decide whether a specific arrow symbol points leftward or rightward (the imperative stimulus). The ANT is designed to examine how different components of attention impact response times. To manipulate the alerting component of attention, the imperative stimulus which otherwise appears after a random time interval is preceded by a visual warning cue to alert the subject that a stimulus is about to appear. The orienting aspects of attention by studied placing the stimulus array above or below the fixation point, and providing or withholding a cue that is spatially predictive, thus allowing the subject to shift spatial attention to the correct location. The executive component of attention is manipulated by introducing or removing conflicting irrelevant information; the imperative stimulus is presented along with "flanking" arrows on either side that either point in the same (congruent) direction or in the conflicting (incongruent) direction. Examining how these three classes of manipulations (alerting, orienting, and conflict) impact response times provides an assay for the efficiency of each of these

${ }^{1}$ As of publication date, Posner and Petersen (1990) has over 2250 Web of Science citations. three components of attention. Given the simplicity and sensitivity of the ANT to three largely independent components of attention, it has been used in children and adults in normal populations, and in cohorts with neuropsychiatric disorders such as ADHD, schizophrenia, and borderline personality disorder (Posner et al., 2002; Rueda et al., 2004; Wang et al., 2005; Roberts et al., 2006; Adolfsdottir et al., 2008; Johnson et al., 2008; Fan et al., 2009).

By approaching the human attention system as one comprised of several separable networks, one can create paradigms to examine how the networks operate in a fairly independent fashion. Alternatively, different paradigms may prove useful in studying the ways in which these systems interact. Using the ANT, early studies of attention networks investigated conditions that emphasized how the networks can be studied as operating independently (Posner and Petersen, 1990; Fan et al., 2002). In fact, in the original study with the ANT, there was statistical independence between manipulations of orienting and conflict, as well as some, but not all, manipulations of alerting. Furthermore, performance scores obtained for each network were uncorrelated, suggesting that individual differences in one component cannot be easily attributed to individual differences in another component, nor attributed to an additional factor that might have influenced multiple networks together. Fan et al. extended the ANT investigation by adapting this general paradigm for use with event-related fMRI (Fan et al., 2005) and joint time-frequency analysis of electroencephalogram (EEG) data (Fan et al., 2007), thus providing further and more direct supporting evidence for the separation of these three attention components into distinct functional networks. Fan et al. (2001) also showed via twin-studies that heritable genetic variation contributes to normal individual differences in executive function presumably via dopamine rich frontal areas like the anterior cingulate, while alerting and orienting performance demonstrated no such heritability. In addition to studies using the ANT, a great deal of research on each of the three attention networks has been conducted that might help constrain our understanding of each network, as well as help generate structural hypotheses for major white matter tract structures that might be involved in these networks.

In the next section we consider such work, including neuroimaging and neuropsychological studies that may help constrain the selection of white matter tracts that may be involved in each of the three attention networks. For this, we shall consider the conflict, alerting, and orienting networks in turn with the aim of identifying white matter tract structures for further investigation for potential structure-function relationships.

Functional and neuropsychological studies have associated performance in conflict tasks (executive control) to the frontal cortex, and more specifically to a network including the anterior cingulate gyrus and lateral prefrontal cortex (Casey et al., 2000; Fan et al., 2002; Rueda et al., 2004; Callejas et al., 2005). The conflict component of attention mediates inhibitory control, resolution of conflicting stimuli impacting decision making, and, in a broader sense, can be considered necessary for decision planning and decision making. This network likely includes white matter tracts that serve to connect these regions with other structures. The frontal lobes contain numerous complex connections with different parts of the brain, many of which pass through the thalamus. As such, it is likely that tracts from the thalamus extending to the frontal lobe 
and anterior cingulated gyrus, such as the ACR, may be part of the executive attention network associated with the conflict component of the ANT. Indeed, a recent study demonstrated that white matter integrity along the left ACR correlated significantly with conflict performance from the ANT in a group of normal adults and also in a cohort of adults with mild traumatic brain injury (Niogi et al., 2008b).

The alerting component of attention is proposed to be responsible for activating the required cognitive systems to make the person ready to respond to a task. This form of phasic alerting is modulated by thalamic, frontal, and parietal regions (Coull et al., 1996; Sturm and Willmes, 2001; Rueda et al., 2004; Callejas et al., 2005; Fan et al., 2005, 2009). Although the reticular activating system is known to be necessary for tonic alertness, it also plays a critical role in phasic alerting (Sturm and Willmes, 2001; Oken et al., 2006; Fan et al., 2009). A likely white matter pathway that connects the proposed regions critically involves the internal capsule. The internal capsule is made up of an anterior limb (ALIC), and posterior limb (PLIC) and the bend between the two limbs referred to as the genu. The ALIC and PLIC directly relay motor and sensory information with ascending and descending fibers between the cerebral cortex and the pyramids of the medulla (Schünke et al., 2007).

The orienting network selects spatial and sensory information. Commonly, this is tested with visual cues indicating the location of an impending target (as in the ANT). The visual orienting system has been associated with brain areas such as the superior and inferior parietal lobes, frontal eye fields, and subcortical areas including the superior colliculus and reticular nuclei in the thalamus (Corbetta et al., 2000, 2002; Fan et al., 2002, 2005, 2007; Callejas et al., 2005; Wang et al., 2005; Himmelbach et al., 2006). In considering white matter tracts likely to modulate the efficiency of the orienting network, the optic radiations (OR) relay visual information via neurons from the lateral geniculate nucleus of the thalamus to the visual cortex. Such geniculate-cortical circuitry has been implicated in attention studies (Schneider and Kastner, 2009). Additionally, the orienting system must relay and compare spatial information from both visual fields which requires connectivity between hemispheres. Lesions studies of the splenium of the corpus callosum (Noudoost et al., 2006), fMRI studies of interhemispheric transfer (Weber et al., 2005), and studies examining callosal thickness in ADHD (Luders et al., 2009) suggest that commissural fibers, particularly the splenium of the corpus callosum, may play a large modulatory role in the function of the visual spatial orienting network.

Prior neuroimaging studies focused primarily on functional activations using fMRI or EEG to isolate the anatomic substrates for the attention networks. These studies have focused on the functional activations (i.e., gray matter) involved in the attention components. It remains unclear what specific white matter pathways modulate each component of attention. As has been demonstrated in several other domains reviewed above, individual differences in white matter microstructure within tracts associated with particular attention networks may closely correlate with variations in efficiency of these attentional processes.

One technique to quantify white matter integrity is DTI (Basser et al., 1994; Basser and Pierpaoli, 1996). The principle governing DTI is that water diffuses more readily along the orientation of axonal fibers than across the fibers due to hindrance from structural elements such as the axolemma and the myelin sheath. The degree of directionality is termed anisotropy. Anisotropy can be measured as the variation in the eigenvalues of the diffusion tensor (Basser et al., 1994; Ulug and van Zijl, 1999). FA, a normalized measure of anisotropy, has been shown to be sensitive to microstructural changes in white matter integrity and organization (Mukherjee and McKinstry, 2006; Niogi et al., 2008a,b). Increasing numbers of DTI studies that correlate FA with cognitive function indicate that such measurements can be used to account for a wide range skills (Moseley et al., 2002; Schmithorst et al., 2002; Medina et al., 2005; Mabbott et al., 2006; Niogi and McCandliss, 2006; Niogi et al., 2008b).

There are a variety of proposed methods to analyze DTI datasets. The current gold standard is to select regions of interest (ROIs) manually either by tracing the structure of interest with a mouse or placing a fixed geometric shaped ROI within the structure. Despite being the gold standard, these techniques are inherently tedious and prone to human error, thus decreasing inter- and intra-rater reliability, especially across laboratories that might adopt different standards and guidelines. An alternative technique of voxel-based analysis (VBA) avoids many of these difficulties, but introduces others. Automated voxel-based techniques first require data to be spatially normalized and then compared on a voxel-by-voxel basis. Thus, the data comparisons are fundamentally probabilistic, and may reflect anomalies or distortions introduced during the spatial normalization procedure (Jones et al., 2005). Studies have suggested that VBA may be inconsistent across specific regions of the brain, particularly in areas with greater anatomical variability (Quarantelli et al., 2002; Tisserand et al., 2002; Ciccarelli et al., 2003). Furthermore, given the complex directional and sensitive nature of DTI data, proper spatial normalization may prove even more difficult than originally realized (Leemans and Jones, 2009). Another difficulty with VBA is that many studies require operating on data sets that have undergone smoothing functions, which may introduce additional limitations on the spatial certainty of results. Despite being a whole-brain automated technique, VBA is prone to variability as well given the complications and non-standardized parameters used in VBA. Jones et al. (2007) conducted a study in which nine different research groups employing their own (and slightly different) voxel-based technique analyzed the same DTI data set to determine the structural difference between patient and control populations. The study showed that each of the nine research groups selected a different area of the brain that differed between the patient and control populations with minimal overlap (Jones et al., 2007).

Still, voxel-based studies are most suitable for identification of unexpected areas of white matter pathology despite the limitations previously mentioned. However, voxel-by-voxel comparisons inherently have power limitations due to the numerous multiple comparisons necessary in this approach. When a priori structures can be identified, a ROI approach can be employed successfully and may be better suited. In this study, we have identified in an a priori fashion, motivated by a literature-based review, a selection of likely white matter tracts to be involved in each component of attention. Thus, in this study we will employ a ROI technique. To counter 
the inter- and intra-rater variability of manual ROI analysis, in the current study an ROI approach employing a semi-automatic segmentation tool called the Reproducible Objective Quantification Scheme (ROQS) (Niogi et al., 2007). A primary benefit of the ROQS analysis is that regions conforming to the boundaries of the tracts are selected using an objective, reproducible algorithm and in a fashion specific to each subject. Unlike VBA, this method does not require spatial normalization of tensor data, thereby operating on the original spatially untransformed data and thus avoiding confounding differences in the size and shape of an individual or template brain. Although a central drawback to this approach is that it does not lend itself to searching the entire brain for a potential correlations, it does provide a limited set of opportunities to examine well constrained $a$ priori hypotheses about a small class of ROIs in white matter tract networks that can be reliably quantified and systematically related to cognitive performance (for a discussion see Niogi et al., 2007).

Here we investigate potential relationships between individual differences in the efficiency of each component of attention and individual differences in FA within specific white matter tract regions. These regions have been selected based on the existing neuroimaging literature reviewed above for the alerting, orienting, and conflict components of attention. Subsequently, we test the specificity of these findings by examining cross-correlation patterns and conducting multiple regression analyses to determine whether each of the three components of attention is related to a specific network.

\section{MATERIALS AND METHODS PARTICIPANTS}

Subjects in this study included 26 healthy adult volunteers ( 19 male, 7 female) with an average age of 28.3 years (range 17-58 years; standard deviation 9.38 years $)^{2}$. Exclusion criteria were imposed for any evidence of abnormal MRI scan, prior history of traumatic brain injury, history of neurological or psychiatric illness including drug or alcohol abuse, psychotropic medications that would affect cognitive testing, or any of a multitude of standard contraindications to MR imaging such as pregnancy or ferromagnetic implants. Written and verbal informed consent was obtained from all subjects in accordance with the Declaration of Helsinki and as approved by the authors' institutions.

\section{ASSESSMENT OF ATTENTION COMPONENT FUNCTION}

The Attention Network Task (Fan et al., 2005) was used to provide a quantitative reaction-time based assessment of each of the three major components of attention. The version of the test employed here was identical to a subset of conditions employed in the Fan et al. (2002) study, including three of the original cue conditions (no cue, central alerting cue, spatial cue) and the original two

\footnotetext{
${ }^{2}$ Twenty-three of these subjects served as normal controls for a separate previously published study investigating the dissociation of attention and memory deficits following chronic mild traumatic brain injury (Niogi et al., 2008b). Please note that the prior investigation (Niogi et al., 2008b) initially reported the findings relating executive attention (conflict) to white matter integrity of the ACR using these 23 subjects. This result is replicated in this current study with the addition of three subjects. While the previous study focused on dissociating attention and memory, it did not provide an in-depth examination of all three components of attention presented here in this current study.
}

target conditions. All trials presented a target stimulus, either above or below the fixation cross. Figure 1 illustrates the various cue and target conditions as well as their timing. All target stimuli included an imperative leftward or rightward pointing arrow centered horizontally above or below the fixation cross, with the direction of the arrow signifying whether the subject was to press the corresponding left or right index finger response key. For all target stimuli, the horizontally centered arrow was flanked on either side by two additional arrows pointing in the congruent or incongruent direction. The entire array of arrows appeared either above or below the fixation cross. Target stimuli were preceded by one of three cue conditions: (1) a no-cue condition which was not perceptively different from the preceding or following fixation stimuli (i.e., baseline, temporally and spatially non-informative), (2) a center-cue condition in which the fixation cross was temporarily replaced by an asterisk appearing directly between the two potential target locations (i.e., temporally informative but spatially uninformative), or (3) a spatial-cue condition in which an asterisk appeared in the exact position of the target arrow (i.e., temporally and spatially informative).

Reaction times were recorded and the difference in reaction times between varying conditions was used to calculate a performance score for each of the three components of attention. Specifically, a conflict score was calculated as the reaction time from all congruent conditions minus the reaction time from all incongruent conditions. Since incongruent trials typically elicit longer reaction times than congruent trials, the conflict score is expected to be negative-valued. A shorter difference in reaction times of the two trials generally indicates better executive control performance and therefore a less negative conflict score. The alerting component of attention was calculated as the reaction times for all no-cue conditions minus reaction times for all center-cue conditions. Finally, the orienting component of attention was calculated as the reaction times from all center cue conditions minus the reaction times for all spatial cue conditions. As the increasingly informative cues are predicted to enhance performance, higher alerting and orienting scores indicate increased efficiency in taking advantage of these specific forms of cue information. As in the Fan et al. (2002) study, obtaining assessments of all three attention components within the very same blocks of trials also provides the opportunity to assess the degree to which each attention component assay is correlated with, or statistically independent, of one another via a simple cross-correlation analysis.

\section{MRI AND DTI ACQUISITION}

Magnetic resonance imaging was acquired on two identical $3 \mathrm{~T}$ GE Signa EXCITE scanners (GE Healthcare, Waukesha, WI, USA) both equipped with the same eight-channel phased-array head coils. Whole-brain DTI was performed with an echoplanar multislice single-shot spin echo pulse sequence $(\mathrm{TE}=63 \mathrm{~ms}, \mathrm{TR}=14 \mathrm{~s})$ using 55 diffusion-encoding directions, isotropically distributed over the surface of a sphere with electrostatic repulsion, acquired at $b=1000 \mathrm{~s} / \mathrm{mm}^{2}, 1$ acquisition with $b=0 \mathrm{~s} / \mathrm{mm}^{2}, 72$ interleaved slices of $1.8-\mathrm{mm}$ thickness each with no gap between slices, a $128 \times 128$ matrix that was zero-filled during reconstruction to $256 \times 256$, and a field of view (FOV) of $230 \mathrm{~mm}$. Parallel imaging was employed using the Array Spatial Sensitivity Encoding 


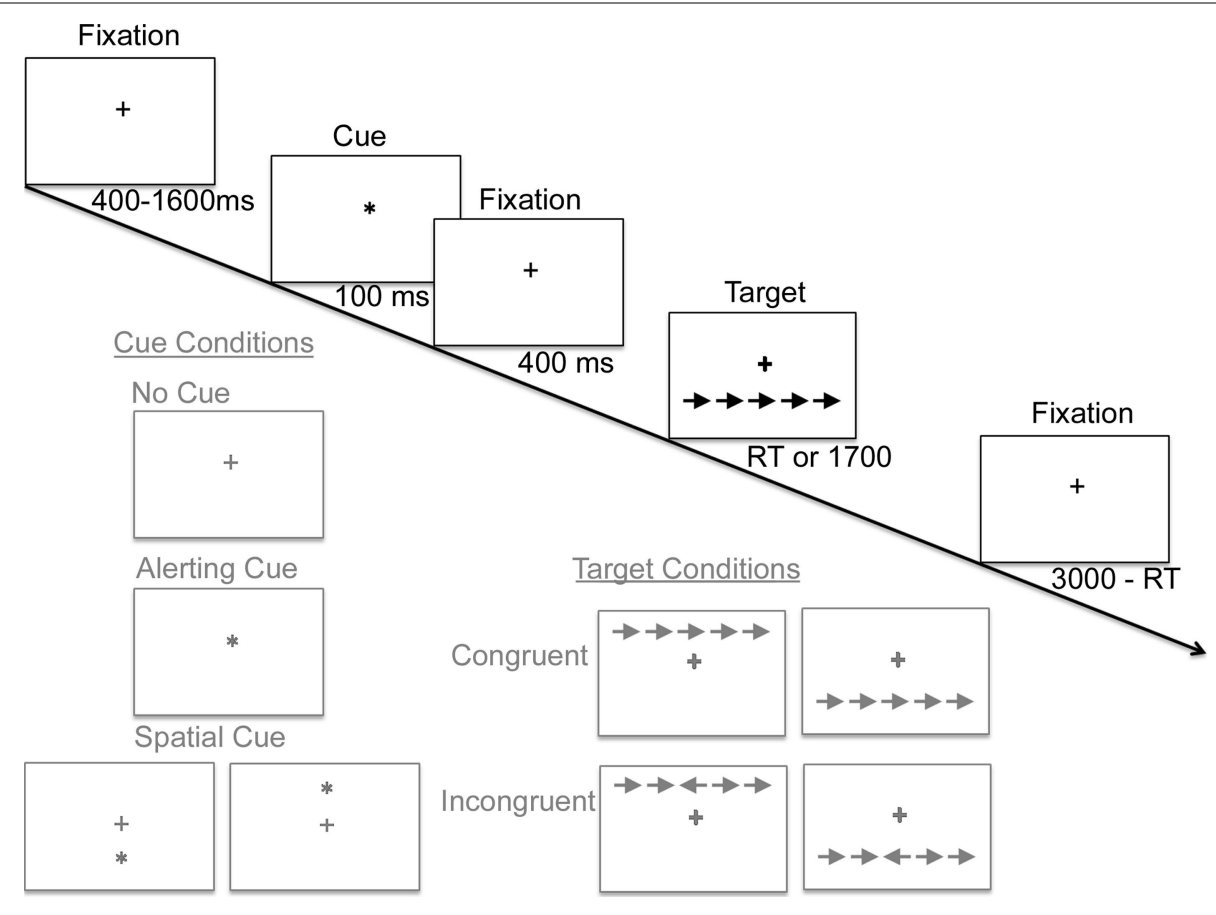

FIGURE 1 | A schematic diagram of a single trial of the Attention Network Task, in which subjects rapidly decide whether the central arrow of an array points leftward or rightward. A fixation cross at the center of the screen remains throughout the trial. After an unpredictable duration, one of three classes of cue conditions is presented (illustrated in gray). Next, following an additional $400 \mathrm{~ms}$ delay, a target display appears until a response is registered or a deadline expires. Sample target displays illustrate (in gray) the $2 \times 2 \times 2$ factorial of the possible arrays of arrows containing a leftward versus rightward central arrow, congruent versus incongruent flanking arrows, and presentation above versus below fixation. The end of each trial, $3000 \mathrm{~ms}$ after target onset, flows seamlessly into the beginning of the next trial.
Technique (ASSET) with an acceleration factor of 2. Images were post-processed offline using DTIstudio software (Jiang et al., 2006) to obtain FA maps, apparent diffusion coefficient (ADC) maps, and directionally encoded color FA maps.

The following conventional $3 \mathrm{~T}$ MR imaging sequences were acquired: (1) axial 3D inversion recovery fast spoiled gradientrecalled echo (FSPGR) T1-weighted images ( TE $=1.5 \mathrm{~ms}$, $\mathrm{TR}=6.3 \mathrm{~ms}, \mathrm{TI}=400 \mathrm{~ms}$, flip angle of $15^{\circ}$ ) with $230 \mathrm{~mm}$ FOV, $1561.0-\mathrm{mm}$ contiguous partitions at a $256 \times 256$ matrix, (2) axial T2-weighted fluid-attenuated inversion recovery (FLAIR) images $(\mathrm{TE}=126 \mathrm{~ms}, \mathrm{TR}=10 \mathrm{~s}, \mathrm{TI}=2200 \mathrm{~ms})$ with $220 \mathrm{~mm}$ FOV, 47-48 $3.0-\mathrm{mm}$ contiguous slices at a $256 \times 256$ matrix, (3) axial magnetization prepared gradient echo (MPGR) $\mathrm{T} 2{ }^{*}$-weighted images $\left(\mathrm{TE}=15 \mathrm{~ms}, \mathrm{TR}=500 \mathrm{~ms}\right.$, flip angle of $\left.20^{\circ}\right)$ with $220 \times 170 \mathrm{~mm}$ FOV, 47-48 $3.0 \mathrm{~mm}$ contiguous slices at a $256 \times 192$ matrix. All conventional MR images were interpreted by attending neuroradiologists certified by the American Board of Radiology to ensure participants had no clinically significant abnormal MR findings.

\section{ROQS ANALYSIS OF INDIVIDUAL NON-TRANSFORMED FA MAPS}

Anatomically defined ROIs were selected and quantified using the ROQS, which is a semi-automated process that segments white matter structures in a way that has been shown to have high inter- and intra-rater reliability (Niogi and McCandliss, 2006; Niogi et al., 2007; van Eimeren et al., 2008). The benefit of the ROQS analysis compared to manual ROI techniques is that a larger region of pixels is selected for quantification in a manner that conforms to the boundaries of a subsection of a white matter tract for each subject in highly individualized fashion that is also objective and highly reproducible. ROQS operates by using directionally encoded information from the principal eigenvector to segment structures within 2D FA maps. ROQS guidelines direct the user to select a representative seed pixel(s) within anatomically specified ROIs. This set of regions is restricted to clearly identifiable white matter tract structures that appear on two-dimensional FA maps as dominated by a single homogenous direction. Contiguous surrounding boundary pixels are selected algorithmically in the same slice of data that share the same diffusion orientation and similar diffusion properties as the seed pixel, based on individual non-normalized DTI data. The final ROI includes the boundary pixels and all the pixels within the boundary (see Niogi et al., 2007 for full methodological details).

\section{REGION OF INTEREST SELECTION}

A highly restricted set of candidate ROIs were selected for each network in an a priori fashion, based on the existing literature regarding the neural networks associated with each of the attention functions assayed in the ANT. Figure 2 illustrates examples of ROQS ROIs for structures analyzed in this investigation (for a better description of the neuroanatomy refer to Mori et al., 2005; Schünke et al., 2007). Given previous results reviewed above associating executive function with FA values in ACR, we restricted selection of ROIs related to the conflict function to the left and right ACR. Prior constraints 


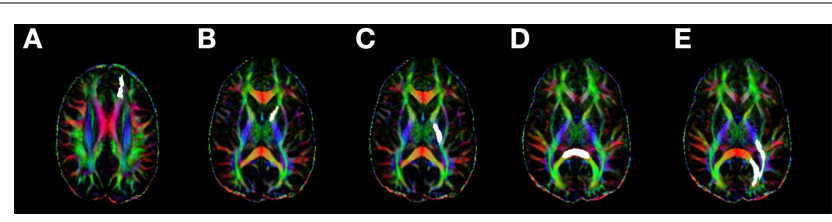

FIGURE 2 |A representative sample of the regions of interest (highlighted in white) measured for each subject using ROQS: (A) anterior corona radiata; (B) anterior limb of the internal capsule; (C) posterior limb of the internal capsule; (D) splenium of the corpus callosum; (E) optic radiations.

regarding the contribution of anterior versus posterior limbs of the internal capsule to phasic alerting functions are somewhat ambiguous, thus both ROIs were chosen bilaterally for investigation of the alerting function (left ALIC, right ALIC, left PLIC, and right PLIC). The splenium of the corpus callosum was investigated as a candidate ROI for orienting on the rationale that this readily identifiable white matter tract structure is involved in the coordination of information across left and right hemisphere posterior parietal regions. Additionally, the OR in each hemisphere were included as candidate ROIs for orienting on the rationale that these regions may mediate connections between thalamic and visual regions involved in processing information from each hemifield.

\section{CONFIRMATORY CORRELATION ANALYSES: PREDICTED STRUCTURES AND FUNCTIONS}

A Cook's $D$ test was used to test for influential data points. This resulted in one subject being excluded from the study for being a statistical outlier. Subsequently, nonparametric statistical tests were then used to assess the amount of structure-function correlation between the ROIs proposed for each attention network and the corresponding ANT behavioral scores. The Spearman's rho statistic was used to test for significance of correlations for each attention function across the set of candidate ROIs proposed for that function, after applying a Bonferroni correction for multiple comparisons (alpha level set to 0.05 divided by the number of candidate ROIs proposed for each attention component).

\section{SPECIFICITY ANALYSES}

\section{Assessing potential cross-correlations across the proposed functional networks}

To assess whether positive findings for structure-function relationships were specific to each network's hypothesized attention function rather than reflecting a systemic relationship between white matter tract properties and cognitive function or specific patterns of shared variance across any two of the functional networks, we first assessed patterns of potential cross-correlations. For this, a reduced set of three candidate ROIs were selected, one for each attention function demonstrating the strongest evidence of a positive relationship. These correlations composed a $3 \times 3$ correlation matrix, with the diagonal representing the predicted set of correlations, and the off-diagonal representing the degree of specificity of those predictions. No corrections were applied to this correlation matrix to avoid any potential type II error bias that would tend to favor the predicted hypothesis of high specificity across attention functions and predicted ROI.

\section{Assessing degree of unique variance accounted for by the proposed functional networks}

The final goal of the study was to test the specificity of the attention components to their corresponding neuronal networks by attempting to establish a triple dissociation. This framework of multiple regression dissociation has proven effective in demonstrating separability of individual difference patterns of structure-function correlations across domains, such as reading versus short term memory (Niogi and McCandliss, 2006) and attention versus memory retrieval (Niogi et al., 2008b). This technique, however, has yet to be applied to dissociating component cognitive processes within a domain. Separability is directly assessed here in a regression framework that incorporates a series of three complementary multiple regression analyses, each with a specific dependent measure (FA in the respective ROIs from the specificity analysis), and three interrelated regressors: subject age in years and the two attention performance measures proposed to be unrelated to the specific functional network under investigation. Finally, the last step of each analysis included the performance scores for the proposed function for the network under investigation after controlling for the other factors.

The aim of these analyses was to assess the unique variance accounted for by each component of attention with the corresponding white matter tract. For each of the three analyses, two blocks of independent variables were applied in a step-wise fashion. The first block of each analysis contained the two attention scores proposed to be irrelevant to that ROI, as well as age in years. The second block added the proposed attention component score. Significance of the $\Delta R^{2}$ change was computed using an $F$ statistic. All statistical tests were performed using SPSS v.14 (SPSS Inc., Chicago, IL, USA).

\section{RESULTS}

\section{BEHAVIORAL CROSS-CORRELATION RESULTS}

Behavioral results were first examined to determine the degree of interdependence of individual differences across the three components of attention. As shown in Table 1 the efficiency scores for each attention component were uncorrelated with one another, replicating findings from Fan et al. (2002) of functional independence across patterns of individual differences for the three components of attention function.

\section{PREDICTED STRUCTURE-FUNCTION CORRELATIONS}

Correlations between conflict scores and FA in the candidate regions of the left and right ACR provided support for this relationship (ACR Left: $r=-0.428, p=0.012$; ACR Right: $r=-0.363, p=0.069$ ). Correlations between alerting scores and FA of the PLIC and ALIC

Table 1 |Attention component correlations.

\begin{tabular}{llll}
\hline & Alerting & Orienting & Conflict \\
\hline Alerting & 1 & $-0.27(p=0.19)$ & $-0.14(p=0.49)$ \\
Orienting & $-0.27(p=0.19)$ & 1 & $0.17(p=0.41)$ \\
Conflict & $-0.14(p=0.48)$ & $0.17(p=0.41)$ & 1 \\
\hline
\end{tabular}

Correlation coefficients between attention component scores. The correlation coefficient is followed by the significance of the correlation (two-tailed) in parenthesis. As seen here, the scores for each component were uncorrelated with one another. 
provided support for a structure-function relationship restricted to the left PLIC (PLIC Left: $r=0.474, p=0.015$; PLIC Right: $r=0.050$, $p=0.81$; ALIC Left: $r=-0.153, p=0.46$; ALIC Right: $r=-0.181$, $p=0.38$ ). Finally, correlations between orienting scores and FA of the splenium of the corpus callosum and the OR provided support for a relationship between the splenium and orienting component (splenium: $r=0.471, p=0.015$; OR Left: $r=0.039, p=0.85$; OR Right: $r=-0.60, p=0.77)$. Before controlling for multiple comparisons across the number of predicted ROIs for each attention func- tion, each of the three attention functions correlate significantly with at least one predicted ROI (see Figure 3). However, after controlling for multiple comparisons using a Bonferroni correction for the set of proposed regions, the conflict-left ACR correlation alpha values is corrected to less than 0.025 ( $p<0.05$ divided by 2 ROIs). The alerting alpha value is corrected to less than $0.0125(p<0.05$ divided by four ROIs), and the orienting component alpha value is corrected to less than 0.017 ( $p<0.05$ divided by three ROIs). Using these criteria, a significant correlation remains between the left ACR

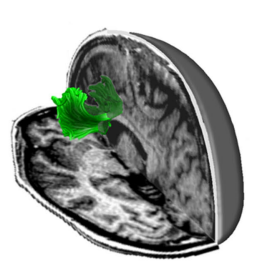

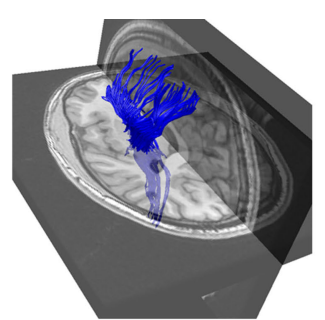

Left Post. Limb of Internal Capsule
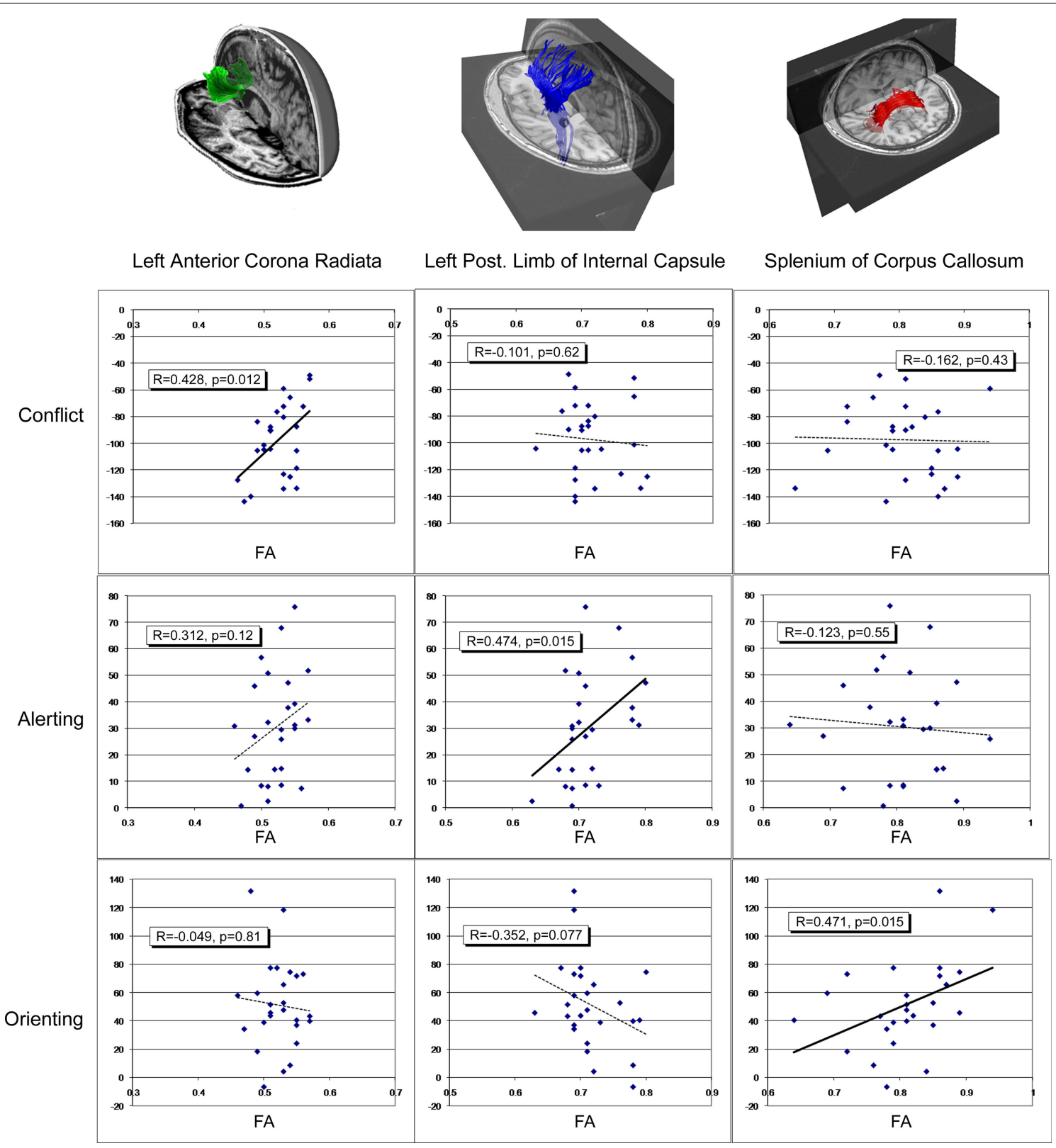

FIGURE 3 | Scatter plots demonstrating the relationship between functional components of attention identified in the Attention Network Task (ANT), and average fractional anisotropy (FA) of relevant regions of interest. Each row of graphs illustrates results for the three components of attention: the conflict component (top row), the alerting component (second row), and the orienting component (third row), each column of graphs illustrates result for a separate structure: the left anterior corona radiata (left column), the left posterior limb of the internal capsule (middle column), and the splenium of the corpus callosum (right column), each of which are illustrated via fiber tracking in the top row. The vertical axis for each graph represents the attention component score in millisecond. The horizontal axis of each graph represents FA of each ROI. 
and conflict scores and between the splenium and orienting scores. The relationship between alerting and the left PLIC fails to pass this more restrictive significance threshold.

\section{SPECIFICITY OF STRUCTURE-FUNCTION RELATIONSHIPS Specificity of structure-function relationships l: assessment of cross-correlations}

Figure 3 illustrates potential patterns of cross-correlation for the three significant structure-function relationships, using uncorrected alpha levels to ensure maximum potential sensitivity to potential cross-talk between the three purportedly functionally specific networks. As predicted, conflict scores failed to correlate with FA in left PLIC $(r=0.101, p=0.624)$ or splenium $(r=0.162, p=0.43)$. Alerting failed to correlate with integrity of the left ACR $(r=0.312$, $p=0.12)$ or splenium $(r=0.123, p=0.55)$. Orienting failed to correlate with integrity of the left ACR $(r=-0.049, p=0.814)$, yet exhibited a non-significant trend toward a correlation with left PLIC $(r=-0.352, p=0.077)$. This trend toward a correlation between orienting and PLIC was further evaluated via the multiple regression analysis (see below).

\section{Specificity of structure-function relationships II: a triple dissociation} A set of three multiple regression analyses (see Tables 2-4) revealed that the proposed relationship between the three attention functions and three corresponding ROIs accounted for unique variance in the predicted structure-function relationships, even after accounting for the potential influences of age and the two purportedly unrelated attention performance measures. In each of the three regression analyses, $R^{2}$ change in the final block indicated that the proposed specific functional scores significantly accounted for unique variance in the analysis of all three ROIs. Specifically, after the addition of conflict in the multiple regression scheme for the left ACR (see Table 2 ) there was a significant $R^{2}$ change $\left(\Delta R^{2}=0.213, p=0.020\right)$ indicating that even after controlling for age and the purportedly irrelevant alerting and orienting scores, conflict still loaded heavily on the left ACR.

Likewise, in the regression analysis of the left PLIC, after the addition of alerting scores (see Table 3 ), there was a significant $R^{2}$ change $\left(\Delta R^{2}=0.15, p=0.05\right)$ indicating PLIC FA scores are uniquely associated with performance in the alerting component of attention even after controlling for age, conflict, and orienting performance.

Finally, after the addition of orienting scores to the analysis of the splenium of the CC (see Table 4), a significant $R^{2}$ change $\left(\Delta R^{2}=0.16, p=0.044\right)$ indicated that specificity of individual differences in FA within the splenium of the CC are specifically related to orienting, above and beyond the potential influence of age or the two other attention functions.

Interestingly, across all three analyses, the combined factors of age and the two purportedly unrelated attention components failed to account for significant variance in any of the ROIs providing additional support for functional independence across these three attention networks.

\section{DISCUSSION}

The attention network framework provides a key hypothesis that the general domain of attentional function, as it is instantiated in the brain, is subserved by three functionally and anatomically separable
Table 2 | Multiple regression analysis for left anterior corona radiata + conflict.

\begin{tabular}{llllll}
\hline Model & $\boldsymbol{R}$ & $\boldsymbol{R}^{\mathbf{2}}$ & $\boldsymbol{R}^{\mathbf{2}}$ change & $\boldsymbol{F}$ change & Sig. F change \\
\hline 1 & $0.291^{\mathrm{a}}$ & 0.084 & 0.084 & 0.676 & 0.576 \\
2 & $0.545^{\mathrm{b}}$ & 0.297 & 0.213 & 6.356 & 0.020
\end{tabular}

a Predictors: (constant), age, orienting, alerting.

${ }^{b}$ Predictors: (constant), age, orienting, alerting, conflict.

Table 3 | Multiple regression analysis for left posterior limb of internal capsule + alerting.

\begin{tabular}{llllll}
\hline Model & $\boldsymbol{R}$ & $\boldsymbol{R}^{\mathbf{2}}$ & $\boldsymbol{R}^{\mathbf{2}}$ change & $\boldsymbol{F}$ change & Sig. F change \\
\hline 1 & $0.359^{\mathrm{a}}$ & 0.129 & 0.129 & 1.087 & 0.375 \\
2 & $0.527^{\mathrm{b}}$ & 0.278 & 0.149 & 4.330 & 0.050 \\
\hline
\end{tabular}

apredictors: (constant), age, orienting, conflict.

bPredictors: (constant), age, orienting, conflict, alerting.

Table 4 | Multiple regression analysis for splenium of corpus callosum + orienting.

\begin{tabular}{llllll}
\hline Model & $\boldsymbol{R}$ & $\boldsymbol{R}^{\mathbf{2}}$ & $\boldsymbol{R}^{\mathbf{2}}$ change & $\boldsymbol{F}$ change & Sig. F change \\
\hline 1 & $0.345^{\mathrm{a}}$ & 0.119 & 0.119 & 0.989 & 0.416 \\
2 & $0.526^{\mathrm{b}}$ & 0.277 & 0.158 & 4.576 & 0.044
\end{tabular}

apredictors: (constant), age, alerting, conflict.

bPredictors: (constant), age, alerting, conflict, orienting.

networks (Posner and Petersen, 1990). Clearly, the three attention networks proposed are functionally and anatomically connected via specific white matter tracts. The central hypotheses investigated in the current study are that individual variation in white matter tract microstructure is systematically linked to individual differences in cognitive efficiency, and that this structure-function relationship plays out in a highly anatomically and functionally specific manner as predicted by the separability hypothesis of the attention network framework.

To show specificity of the findings we employed a correlational dissociation (Niogi and McCandliss, 2006; Niogi et al., 2008b), which is akin to neuropsychological lesion studies. Instead of using a lesion to show specificity by loss of function, the correlational dissociation used a series of multiple regression analyses to show specificity of structure and function by correlating integrity and performance. The correlational triple dissociation has similar benefits and limitations as lesions studies. Just as a lesion study cannot make claims that other structures may be necessary or involved with the lost function, the correlational triple dissociation we employed does not claim that regions outside the ones analyzed are not involved in attention. The benefit of the correlational dissociation is that we showed that the structures examined are involved in the attention network and that, among the structures analyzed, each tract has component specificity. To establish the correlational dissociation, three criteria (similar to the criteria for a lesion study) were needed and were met. First, a control region is necessary to show specificity. This was achieved because all three 
ROIs (ACR, PLIC, and splenium) correlated with one cognitive process. Secondly, the correlation must remain significant after controlling for the other domains (this was shown successfully using the multiple regression analyses). Finally, the structure-function relationship demonstrated a lack of correlation with the two other control structures (see Figure 3).

At the most general level, the overall pattern of results provides support for the central hypothesis that distinct white matter tract networks exist for each component of attention. In order to test these hypotheses it was first necessary to propose candidate ROIs that might uniquely capture each functional network based on existing literature and empirically establish whether any subset of these candidate ROIs related to the proposed functions. FA values from a subset of the candidate ROIs demonstrated a set of network specific brain-behavior correlations for each of the three attention components. Each of these positive correlations suggests that white matter tract microstructure accounts for substantial variance in the efficiency of attention performance. Next, examination of the cross-correlation patterns of these three positive findings of structure-function relationships revealed a dramatic pattern of specificity, suggesting that none of the three positive correlations were readily attributable to the influence of an additional common factor. Finally, the multiple regression dissociation analysis provided further evidence and critical support for the specificity hypothesis that each of the proposed structure-function relationships was quite specific in nature and accounted for unique variance even after the influence of age and the purported non-related attention components were taken into account.

Next, we consider the evidence and implications for each network, and then turn to the central question of the functional and anatomical separability of the three component networks of attention.

\section{THE CONFLICT NETWORK}

The correlation analysis together with the multiple regression dissociation analysis provides evidence that microstructural integrity of the ACR modulates executive attention. The finding of a frontal tract associated with executive attention is strongly supported by previous literature. Functional imaging studies have provided strong consistent support for the notion that the frontal lobes, particularly the anterior cingulate gyrus, is associated with attentional control during the Stroop Task and the conflict portion of the Attention Network Task (Casey et al., 2000; Gruber et al., 2002; Fan et al., 2005). Additionally, neuropsychological data clearly highlight the central role of the frontal lobes in executive attention (Stuss et al., 1981; Stuss and Benson, 1984; Vendrell et al., 1995; Stuss and Alexander, 2000). Previous DTI studies have also implicated frontal white matter tracts with attention performance. For example, integrity of white matter tracts associated with brain activity impacts executive attention (Olesen et al., 2003; Madden et al., 2006). Furthermore, Niogi and McCandliss (2006) demonstrated that a closely related frontal executive skill, short term memory, also demonstrated strong correlations with ACR in children. Finally, in a recent study of 43 patients suffering from mild traumatic brain injury, the left ACR integrity assessed by FA was shown to correlate with conflict scores on the ANT (Niogi et al., 2008b). It is interesting to note that in the current study, significant correlation between conflict scores and FA did not reach significance in the right ACR, but appeared only as a non-significant trend. Given this trend and no significant finding of a direct effect of laterality, it is possible that both left and right ACR regions play a role in conflict. The specific contributions of right ACR may be apparent in studies with greater power.

\section{THE ORIENTING NETWORK}

Regarding the orienting network, the correlation analysis together with the multiple regression dissociation analysis demonstrates that microstructural integrity of the splenium of the corpus callosum modulates the efficiency of the orienting component of attention. This finding is consistent with previous findings that demonstrate that the visual spatial attentional orienting system is dependent on a functional network that includes left and right posterior parietal regions and is linked to a larger neural network including frontal eye fields, subcortical areas including the superior colliculus, and reticular nuclei in the thalamus (Corbetta et al., 2000, 2002; Fan et al., 2002, 2005, 2007; Wang et al., 2005; Himmelbach et al., 2006). We propose that the splenium of the corpus callosum includes white matter tracts involved in this overall network, and that this region provides a large convenient homogenous white matter region of interest that can be used to assess individual differences in this network. Our more specific findings implicating the splenium of the corpus callosum as part of the network that modulates orienting performance is consistent with Noudoost et al.'s (2006) report of a case study that demonstrates the role of interhemispheric connections in making an integral visual map across hemifields that can be used for visual spatial attention. Furthermore, splenium of the corpus callosum lesions commonly cause visual spatial neglect (Tassinari et al., 1994; Park et al., 2005; Noudoost et al., 2006), a deficit that specifically impacts orienting functions of attention. Such lesions can also cause other forms of disconnection syndromes that may be linked to spatial orienting functions as in the case or hemialexia (Sugishita et al., 1986; Suzuki et al., 1998; Le et al., 2005) and visual attention (Mayer et al., 1988).

\section{THE ALERTING NETWORK}

The correlation analysis together with the multiple regression dissociation analysis provides evidence that the PLIC modulates individual differences in the alerting component of attention. This relationship is generally consistent with the previous literature suggesting a role of the PLIC for this component of attention. For example, Sturm et al. (1999) presented PET evidence from normal volunteers that suggested alerting functions involve a vast network of regions, including inferior parietal-thalamic networks, brainstem structures, and frontal regions largely lateralized to the right hemisphere. Fimm et al. (2001) investigated alerting-related impairments of attention in 15 patients with acute circumscribed vascular lesions confined to the basal ganglia, internal capsule, and thalamus. In his study, five out of seven patients showed evidence of lesions to the posterior limb of the internal capsule. Fimm et al. (2001) suggested that thalamo-parietal projections transversing through the PLIC via the superior peduncle of the thalamus could lead to a disconnection of functionally relevant structures that impair attention. Curiously, the laterality of the PLIC-alerting effects in the present study runs counter to patterns of laterality in lesion studies. While the majority of alerting deficit findings reported above show a bias toward right 
sided lesions linked to loss of attention functions associated with alerting, at least some findings suggest that left PLIC may play a role. For example, Fimm et al. (2001) reported two patients presenting with left hemisphere PLIC lesions, each demonstrating deficits in alerting-related attention skills. Although it is possible that laterality of PLIC plays out differently in the modulation of individual differences in normal control subjects and patients with rather severe damage to these structures, it is also important to note that the pattern of results reported here do not implicate significant lateralization effects per se, but rather demonstrate higher sensitivity to individual difference in alerting in the left PLIC.

\section{FUNCTIONAL AND ANATOMICAL SEPARABILITY OF ATTENTION NETWORKS}

Next, we turn consideration to the separability hypothesis regarding the three attentional networks. In evaluating these specificity findings, it is important to consider that the ANT was specifically designed to stress the potential conditions under which the three attention networks could be shown to be separable in their operations and individual difference patterns. More recently, several investigations have begun to explore other conditions under which these networks might interact and influence one another in important ways (for a review see Fan et al., 2009). To the degree to which the ANT exposes these networks to be anatomically and functionally separable, it is possible that white matter tract integrity is an important independent modulator of function such that individual differences in white matter tract microstructure in one network might be functionally associated with one component of attention relative to the other components of attention. This hypothesis was addressed by a series of multiple regressions in which the variance in function for each established white matter tract ROI was assessed for the key proposed associated function while controlling for the influence of the other component attentional functions.

Results of this multiple regression analysis demonstrated three key findings: the ACR is a unique modulator (relative to the other two ROIs) of the conflict network function, the PLIC is a unique modulator of the alerting network, and the splenium is a unique modulator of the orienting network. This correlational triple dissociation can be interpreted similarly to the "gold-standard" double-dissociation tests in neuropsychology commonly required when attempting to establish specificity of structure-function relationships. Such findings are critical in lesion studies in which the mere presence of any form of brain damage can cause widespread, non-specific difficulties in any performance assessment of cognitive function, and establishing specificity of functional and structural loss is critical. Similarly, in linking individual differences between white matter properties and cognitive performance, establishing similarly high levels of specificity between particular regions and particular functions is critical.

One non-significant trend in the cross-correlation analysis suggested a potential exception to the separability of the three networks. The trend toward correlation between the PLIC and orienting component runs counter to the specificity hypothesis that separate networks are associated with orienting and alerting functions. Critically, this trend only appeared in the zero-order correlational analysis. Further investigation of these phenomena was available from the multiple regression dissociation analysis of the PLIC. By first regressing age, alerting, and conflict on the results, this analysis demonstrated that alerting accounts for unique variance after controlling for the potential influences of other attention factors.

In addition to showing functional specificity, the correlational dissociation analysis shows that within each functional network, a gradient of individual differences in function positively correlates with a gradient of individual differences in white matter tract microstructure. Furthermore, since this study focused on normal healthy adults, rather than reflecting the impact of neural damage on related loss of function, the results may be more relevant to understanding normal population variations in white matter tract microstructure and how such differences are linked to efficiency of components of attention. As such, these findings may provide the basis of relating dimensional differences in structure-function relationships that exist within normal populations with more extreme ranges of variation of structural damage and related loss of function (for discussion see Niogi and McCandliss, 2006).

\section{FURTHER CONSIDERATIONS}

It is important to note that the ROI approach adopted here was motivated by a theoretical assumption that average FA taken in one region of a specific network may provide an index of white matter tract properties shared with other parts of this network. Claims of specificity are not meant to imply that this ROI is the only region of a network sharing these properties, but rather that this region of this network can be functionally differentiated from other regions of other networks. Furthermore, the technique used to define ROIs in this study, the ROQS method, avoids any form of spatial transformation to the DTI FA data by defining each ROI on a subject-by-subject basis.

There are a few additional limitations of this study that may be addressed in future investigations of white matter pathways involved in the components of the attention network. First, this study was a preliminary study for which it was necessary to explore a small set of ROIs that was motivated by previous literature findings and the insights of the attentional networks implicated from pervious imaging and neuropsychological studies. On one hand small set of regions explored for each function lead to fewer multiple comparison corrections and a better-powered analysis. On the other hand, only a small fraction of each network was included in each ROI, and thus the majority of white matter tracts within each network were left unexplored. The small sample size might have also limited insights into the functional significance of laterality results, such as the involvement of the right hemisphere ACR to the conflict network and the lateralization of the alerting network. Additionally, the study focused on which white matter networks are directly involved in each component. If we are to believe that there must be some sort of interaction between component networks (in addition to being individually and independently modulated by the proposed pathways), then it is possible in future studies to examine the connectivity between the white matter pathways. Future studies may benefit from combining fMRI with DTI as well as implementing tractography to study the connections between networks. Finally, the nature of the ROI approach 
adopted in this study is inherently limited to a priori predictions of regions that might be implicated in function. Exploratory analyses examining white matter tract regions throughout the brain may play an important role in providing new hypotheses to test in future studies.

In conclusion, combining these observations with previous fMRI and neuropsychological studies suggests the components of attention are comprised of segregated functional networks and that individual differences in white matter tract microstructural integrity might modulate these functionally specific neural networks. Thus, understanding individual differences in white matter tract microstructural integrity may prove an important complement to fMRI studies of functional and anatomical organization of attention.

\section{ACKNOWLEDGMENTS}

This work was supported by the James S. McDonnell Foundation through a collaborative grant to the Brain Trauma Foundation and by a National Science Foundation grant to author Bruce D. McCandliss (REC-0337715). Special thanks to R. Kolster and R. Sarkar for help in acquiring ANT behavioral data.

\section{REFERENCES}

Adolfsdottir, S., Sorensen, L., and Lundervold,A. J. (2008). The attention network test: a characteristic pattern of deficits in children with ADHD. Behav. Brain Funct. 4, 9.

Basser, P. J., Mattiello, J., and LeBihan, D. (1994). MR diffusion tensor spectroscopy and imaging. Biophys. J. 66, 259-267.

Basser, P. J., and Pierpaoli, C. (1996). Microstructural and physiological features of tissues elucidated by quantitative-diffusion-tensor MRI. J. Magn. Reson. B 111, 209-219.

Beaulieu, C., Plewes, C., Paulson, L.A., Roy, D., Snook, L., Concha, L., and Phillips, L. (2005). Imaging brain connectivity in children with diverse reading ability. Neuroimage 25, 1266-1271.

Callejas, A., Lupianez, J., Funes, M. J., and Tudela, P. (2005). Modulations among the alerting, orienting and executive control networks. Exp. Brain Res. 167, 27-37.

Casey, B. J., Thomas, K. M., Welsh, T. F., Badgaiyan, R. D., Eccard, C. H., Jennings, J. R., and Crone, E. A. (2000). Dissociation of response conflict, attentional selection, and expectancy with functional magnetic resonance imaging. Proc. Natl. Acad. Sci. U.S.A. 97, 8728-8733.

Ciccarelli, O., Werring, D. J., Barker, G. J., Griffin, C. M., Wheeler-Kingshott, C. A., Miller, D. H., and Thompson, A. J. (2003). A study of the mechanisms of normal-appearing white matter damage in multiple sclerosis using diffusion tensor imaging - evidence of Wallerian degeneration. J. Neurol. 250, 287-292.

Corbetta, M., Kincade, J. M., Ollinger, J. M., McAvoy, M. P., and Shulman, G. L. (2000). Voluntary orienting is dissociated from target detection in human posterior parietal cortex. Nat. Neurosci. 3, 292-297.

Corbetta, M., Kincade, J.M., and Shulman, G. L. (2002). Neural systems for visual orienting and their relationships to spatial working memory. J. Cogn. Neurosci. 14, 508-523.

Coull, J. T., Frith, C. D., Frackowiak, R. S., and Grasby, P. M. (1996). A fronto-parietal network for rapid visual information processing: a PET study of sustained attention and working memory. Neuropsychologia 34, 1085-1095.

Deutsch, G. K., Dougherty, R. F., Bammer, R., Siok, W. T., Gabrieli, J. D., and Wandell, B. (2005). Children's reading performance is correlated with white matter structure measured by diffusion tensor imaging. Cortex 41, 354-363.

Dougherty, R. F., Ben-Shachar, M., Deutsch, G. K., Hernandez, A., Fox, G. R., and Wandell, B. A. (2007). Temporal-callosal pathway diffusivity predicts phonological skills in children. Proc. Natl. Acad. Sci. U.S.A. 104, 8556-8561.

Ewing-Cobbs, L., Hasan, K. M., Prasad, M. R., Kramer, L., and Bachevalier, J. (2006). Corpus callosum diffusion anisotropy correlates with neuropsychological outcomes in twins disconcordant for traumatic brain injury. AJNR Am. J. Neuroradiol. 27, 879-881.

Fan, J., Byrne, J., Worden, M. S., Guise, K. G., McCandliss, B. D., Fossella, J., and Posner, M. I. (2007). The relation of brain oscillations to attentional networks. J. Neurosci. 27, 6197-6206.

Fan, J., Gu, X., Guise, K. G., Liu, X., Fossella, J., Wang, H., and Posner, M. I. (2009). Testing the behavioral interaction and integration of attentional networks. Brain Cogn. 70, 209-220.

Fan, J., McCandliss, B. D., Fossella, J., Flombaum, J. I., and Posner, M. I. (2005). The activation of attentional networks. Neuroimage 26, 471-479.

Fan, J., McCandliss, B. D., Sommer, T., Raz, A., and Posner, M. I. (2002). Testing the efficiency and independence of attentional networks. J. Cogn. Neurosci. 14, 340-347.

Fan, J., Wu, Y., Fossella, J. A., and Posner, M. I. (2001). Assessing the heritability of attentional networks. BMC Neurosci. 2, 14.

Fimm, B., Zahn, R., Mull, M., Kemeny, S., Buchwald, F., Block, F., and Schwarz,M. (2001). Asymmetries of visual attention after circumscribed subcortical vascular lesions. J. Neurol. Neurosurg. Psychiatr. 71, 652-657.

Grieve, S. M., Williams, L. M., Paul, R. H., Clark, C. R., and Gordon, E. (2007). Cognitive aging, executive function, and fractional anisotropy: a diffusion tensor MR imaging study. AJNR Am. J. Neuroradiol. 28, 226-235.

Gruber, S. A., Rogowska, J., Holcomb, P., Soraci, S., and Yurgelun-Todd, D (2002). Stroop performance in normal control subjects: an fMRI study. Neuroimage 16, 349-360.

Himmelbach, M., Erb, M., and Karnath, $\mathrm{H}$. O. (2006). Exploring the visual world: the neural substrate of spatial orienting. Neuroimage 32, 1747-1759.

Jiang, H., van Zijl, P. C., Kim, J., Pearlson, G. D., and Mori, S. (2006). DtiStudio: resource program for diffusion tensor computation and fiber bundle tracking. Comput. Methods Programs Biomed. 81, 106-116.

Johnson, K. A., Robertson, I. H., Barry, E., Mulligan, A., Daibhis, A., Daly, M., Watchorn, A., Gill, M., and Bellgrove, M. A. (2008). Impaired conflict resolution and alerting in children with ADHD: evidence from the Attention Network Task (ANT). J. Child. Psychol. Psychiatry 49, 1339-1347.

Jones, D. K., Chitnis, X. A., Job, D., Khong, P. L., Leung, L. T., Marenco, S., Smith, S. M., and Symms, M. R. (2007) What happens when nine different groups analyze the same DT-MRI data set using voxel-based methods? In Proceedings of the 15th Annual Meeting of the International Society for Magnetic Resonance in Medicine, Berlin, p. 74.

Jones, D. K., Symms, M. R., Cercignani, M., and Howard, R. J. (2005). The effect of filter size on VBM analyses of DT-MRI data. Neuroimage 26 546-554.

Klingberg, T., Hedehus, M., Temple, E., Salz, T., Gabrieli, J. D., Moseley, M. E., and Poldrack, R. A. (2000). Microstructure of temporo-parietal white matter as a basis for reading ability: evidence from diffusion tensor magnetic resonance imaging. Neuron $25,493-500$.
Le, T. H., Mukherjee, P., Henry, R. G., Berman, J. I., Ware, M., and Manley, G. T. (2005). Diffusion tensor imaging with three-dimensional fiber tractography of traumatic axonal shearing injury: an imaging correlate for the posterior callosal "disconnection" syndrome: case report. Neurosurgery 56, 189.

Leemans, A., and Jones, D. K. (2009). The $\mathrm{B}$-matrix must be rotated when correcting for subject motion in DTI data. Magn. Reson. Med. 61, 1336-1349.

Liston, C., Watts, R., Tottenham, N., Davidson, M.C., Niogi, S., Ulug, A. M., and Casey, B. J. (2006). Frontostriatal microstructure modulates efficient recruitment of cognitive control. Cereb. Cortex 16, 553-560.

Luders, E., Narr, K. L., Hamilton, L. S., Phillips, O. R., Thompson, P. M., Valle, J.S., Del'Homme, M., Strickland, T., McCracken, J. T., Toga, A. W., McCracken, J. T., and Levitt, J. G. (2009). Decreased callosal thickness in attention-deficit/hyperactivity disorder. Biol. Psychiatry 65, 84-88.

Mabbott, D. J., Noseworthy, M., Bouffet, E., Laughlin, S., and Rockel, C. (2006). White matter growth as a mechanism of cognitive development in children. Neuroimage 33, 936-946.

Madden, D. J., Spaniol, J., Whiting, W. L., Bucur, B., Provenzale, J. M., Cabeza, R., White, L. E., and Huettel, S. A. (2006). Adult age differences in the functional neuroanatomy of visual attention: a combined fMRI and DTI study. Neurobiol. Aging. 28, 459-476.

Mayer, E., Koenig, O., and Panchaud, A. (1988). Tactual extinction without anomia: evidence of attentional factors in a patient with a partial callosal disconnection. Neuropsychologia 26, 851-868.

Medina, D., Detoledo-Morrell, L. Urresta, F., Gabrieli, J. D., Moseley, M., Fleischman, D., Bennett, D. A., Leurgans, S., Turner, D. A., and Stebbins, G. T. (2005). White matter changes in mild cognitive impairment and AD: a diffusion tensor imaging study. Neurobiol. Aging. 27, 663-672.

Mori, S., Wakana, S., and Van Zijl, P. C. M. (2005). MRI Atlas of Human White 
Matter, 1st Edn. Amsterdam/San Diego, CA, Elsevier.

Moseley, M., Bammer, R., and Illes, J. (2002). Diffusion-tensor imaging of cognitive performance. Brain Cogn. 50, 396-413.

Mukherjee, P., and McKinstry, R. C. (2006). Diffusion tensor imaging and tractography of human brain development. Neuroimaging Clin. N. Am. 16, 19-43, vii.

Nestor, P. G., Kubicki, M., Spencer, K. M., Niznikiewicz, M., McCarley, R.W., and Shenton, M. E. (2007). Attentional networks and cingulum bundle in chronic schizophrenia. Schizophr. Res. 90, 308-315.

Niogi, S. N., and McCandliss, B. D. (2006). Left lateralized white matter microstructure accounts for individual differences in reading ability and disability. Neuropsychologia 44, 2178-2188.

Niogi, S. N., Mukherjee, P., Johnson, C., Kolster, R. A., Sarkar, R., Lee, H., Meeker, M., Zimmerman, R. D., Manley, G. T., and McCandliss, B. D. (2008a). Extent of microstructural white matter injury in postconcussive syndrome correlates with impaired cognitive reaction time: a $3 \mathrm{~T}$ diffusion tensor imaging study of mild traumatic brain injury. AJNR Am. J. Neuroradiol. 29, 967-973.

Niogi, S. N., Mukherjee, P., Ghajar, J., Johnson, C. E., Kolster, R., Lee, H., Suh, M., Zimmerman, R. D., Manley, G. T., and McCandliss, B. D. (2008b). Structural dissociation of attentional control and memory in adults with and without mild traumatic brain injury. Brain 131, 3209-3221.

Niogi,S.N.,Mukherjee,P., and McCandliss, B. D. (2007). Diffusion tensor imaging segmentation of white matter structures using a Reproducible Objective Quantification Scheme (ROQS). Neuroimage 35, 166-174.

Noudoost, B.,Afraz, S. R., Vaziri-Pashkam, M., and Esteky, H. (2006). Visual spatial integrity in the absence of splenium. Brain Res. 1076, 177-186.

Odegard, T. N., Farris, E. A., Ring, J., McColl, R., and Black, J. (2009). Brain connectivity in non-reading impaired children and children diagnosed with developmental dyslexia. Neuropsychologia 47, 1972-1977.

Oken, B. S., Salinsky, M. C., and Elsas, S. M. (2006). Vigilance, alertness, or sustained attention: physiological basis and measurement. Clin. Neurophysiol. 117, 1885-1901.

Olesen, P. J., Nagy, Z., Westerberg, H., and Klingberg, T. (2003). Combined analysis of DTI and fMRI data reveals a joint maturation of white and grey matter in a fronto-parietal network. Brain Res. Cogn. Brain Res. 18, 48-57.

Park, K. C., Jeong, Y., Lee, B. H., Kim, E. J., Kim, G. M., Heilman, K. M., and Na, D. L. (2005). Left hemispatial visual neglect associated with a combined right occipital and splenial lesion: another disconnection syndrome. Neurocase 11, 310-318.

Posner, M. I., and Petersen, S. E. (1990). The attention system of the human brain. Annu. Rev. Neurosci. 13, 25-42.

Posner, M. I., and Rothbart, M. K. (2006). Research on attention networks as a model for the integration of psychological science. Annu. Rev. Psychol. $58,1-23$.

Posner, M. I., Rothbart, M. K., Vizueta, N., Levy, K. N., Evans, D. E., Thomas, K. M., and Clarkin, J. F. (2002). Attentional mechanisms of borderline personality disorder. Proc. Natl. Acad. Sci. U.S.A. 99, 16366-16370.

Posner, M. I., Sheese, B. E., Odludas, Y. and Tang, Y. (2006). Analyzing and shaping human attentional networks. Neural Netw. 19, 1422-1429.

Qiu, D., Tan, L.H., Zhou, K., and Khong, P. L. (2008). Diffusion tensor imaging of normal white matter maturation from late childhood to young adulthood: voxel-wise evaluation of mean diffusivity, fractional anisotropy, radial and axial diffusivities, and correlation with reading development. Neuroimage 41, 223-232.

Quarantelli, M., Larobina, M., Volpe, U., Amati, G., Tedeschi, E., Ciarmiello, A., Brunetti, A., Galderisi, S., and Alfano, B. (2002). Stereotaxy-based regional brain volumetry applied to segmented MRI: validation and results in deficit and nondeficit schizophrenia. Neuroimage 17, 373-384.

Raz, A., and Buhle, J. (2006). Typologies of attentional networks. Nat. Rev. Neurosci. 7, 367-379.

Roberts, K. L., Summerfield, A. Q., and Hall, D.A. (2006). Presentation modality influences behavioral measures of alerting, orienting, and executive control. J. Int. Neuropsychol. Soc. 12, 485-492.

Rueda, M. R., Fan, J., McCandliss, B D., Halparin, J. D., Gruber, D. B., Lercari, L. P., and Posner, M. I. (2004). Development of attentional networks in childhood. Neuropsychologia 42, 1029-1040.

Schiavone, F., Charlton, R. A., Barrick, T. R., Morris, R. G., and Markus, H. S (2009). Imaging age-related cognitive decline: a comparison of diffusion tensor and magnetization transfer MRI. J. Magn. Reson. Imaging 29, 23-30.

Schmithorst, V. J., Wilke, M., Dardzinski, B. J., and Holland, S. K. (2002).
Correlation of white matter diffusivity and anisotropy with age during childhood and adolescence: a cross-sectional diffusion-tensor MR imaging study. Radiology 222, 212-218.

Schneider, K. A., and Kastner, S. (2009). Effects of sustained spatial attention in the human lateral geniculate nucleus and superior colliculus. J. Neurosci. 29 , 1784-1795.

Schünke, M., Ross, L. M., Lamperti, E. D., Schulte, E., and Schumacher, U. (2007). Head and Neuroanatomy. Stuttgart/New York, Thieme.

Sturm, W., de Simone, A., Krause, B. J., Specht, K., Hesselmann, V. Radermacher, I., Herzog, H., Tellmann, L., Muller-Gartner, H. W., and Willmes, K. (1999). Functional anatomy of intrinsic alertness: evidence for a fronto-parietal-thalamic-brainstem network in the right hemisphere. Neuropsychologia 37, 797-805.

Sturm, W., and Willmes, K. (2001). On the functional neuroanatomy of intrinsic and phasic alertness. Neuroimage 14 S76-84.

Stuss, D. T., and Alexander, M. P. (2000) Executive functions and the frontal lobes: a conceptual view. Psychol. Res. 63, 289-298.

Stuss, D. T., and Benson, D. F. (1984) Neuropsychological studies of the frontal lobes. Psychol. Bull. 95, 3-28.

Stuss, D. T., Kaplan, E. F., Benson, D. F., Weir, W. S., Naeser, M. A., and Levine, H. L. (1981). Long-term effects of prefrontal leucotomy - an overview of neuropsychologic residuals. J. Clin. Neuropsychol. 3, 13-32.

Sugishita, M., Yoshioka, M., and Kawamura, M. (1986). Recovery from hemialexia. Brain Lang. 29, 106-118.

Suzuki, K., Yamadori, A., Endo, K., Fujii, T., Ezura, M., and Takahashi,A. (1998). Dissociation of letter and picture naming resulting from callosal disconnection. Neurology 51, 1390-1394.

Tassinari, G., Aglioti, S., Pallini, R., Berlucchi, G., and Rossi, G. F. (1994) Interhemispheric integration of simple visuomotor responses in patients with partial callosal defects. Behav. Brain Res. 64, 141-149.

Tisserand, D. J., Pruessner, J. C., Sanz Arigita, E. J., van Boxtel, M. P., Evans, A. C., Jolles, J., and Uylings, H. B. (2002). Regional frontal cortical volumes decrease differentially in aging: an MRI study to compare volumetric approaches and voxel-based morphometry. Neuroimage 17, 657-669.

Tuch, D. S., Salat, D. H., Wisco, J. J., Zaleta A. K., Hevelone, N. D., and Rosas, H. D. (2005). Choice reaction time performance correlates with diffusion anisotropy in white matter pathways supporting visuospatial attention. Proc. Natl. Acad. Sci. U.S.A. 102, 12212-12217.

Ulug, A. M., and van Zijl, P. C. (1999). Orientation-independent diffusion imaging without tensor diagonalization: anisotropy definitions based on physical attributes of the diffusion ellipsoid. J. Magn. Reson. Imaging 9, 804-813.

van Eimeren, L., Niogi, S. N., McCandliss, B. D., Holloway, I. D., and Ansari, D. (2008). White matter microstructures underlying mathematical abilities in children. Neuroreport 19, 1117-1121.

Vendrell, P., Junque, C., Pujol, J., Jurado, M. A., Molet, J., and Grafman, J. (1995). The role of prefrontal regions in the Stroop task. Neuropsychologia 33, 341-352.

Wang, K., Fan, J., Dong, Y., Wang, C. Q., Lee, T. M., and Posner, M. I. (2005). Selective impairment of attentional networks of orienting and executive control in schizophrenia. Schizophr. Res. 78, 235-241.

Weber, B., Treyer, V., Oberholzer, N., Jaermann, T., Boesiger, P., Brugger, P., Regard, M., Buck, A., Savazzi, S., and Marzi, C. A. (2005). Attention and interhemispheric transfer: a behavioral and fMRI study. J. Cogn. Neurosci. 17, 113-123.

Zahr, N. M., Rohlfing, T., Pfefferbaum, A., and Sullivan, E. V. (2009). Problem solving, working memory, and motor correlates of association and commissural fiber bundles in normal aging: a quantitative fiber tracking study. Neuroimage 44, 1050-1062.

Conflict of Interest Statement: The authors declare that the research was conducted in the absence of any commercial or financial relationships that could be construed as a potential conflict of interest.

Received: 07 July 2009; paper pending published: 05 August 2009; accepted: 05 January 2010; published online: 22 February 2010

Citation: Niogi S, Mukherjee P, Ghajar J and McCandliss BD (2010) Individual differences in distinct components of attention are linked to anatomical variations in distinct white matter tracts. Front. Neuroanat. 4:2. doi: 10.3389/neuro.05.002.2010 Copyright (c) 2010 Niogi, Mukherjee, Ghajar and McCandliss. This is an openaccess article subject to an exclusive license agreement between the authors and the Frontiers Research Foundation, which permits unrestricted use, distribution, and reproduction in any medium, provided the original authors and source are credited. 